\title{
On Deformation of Foliations with a Center in the Projective Space
}

\author{
HOSSEIN MOVASATI \\ IMPA - Instituto de Matemática Pura e Aplicada \\ Estrada Dona Castorina, 110 - 22460-320 Rio de Janeiro, RJ, Brazil. \\ Manuscript received on January 31, 2001; accepted for publication on February 14, 2001; \\ presented by A. Lins NETO
}

\begin{abstract}
Let $\mathcal{F}$ be a foliation in the projective space of dimension two with a first integral of the type $\frac{F^{p}}{G^{q}}$, where $F$ and $G$ are two polynomials on an affine coordinate, $\frac{\operatorname{deg}(F)}{\operatorname{deg}(G)}=\frac{q}{p}$ and $g . c . d .(p, q)=1$. Let $z$ be a nondegenerate critical point of $\frac{F^{p}}{G^{q}}$, which is a center singularity of $\mathcal{F}$, and $\mathcal{F}_{t}$ be a deformation of $\mathcal{F}$ in the space of foliations of degree $\operatorname{deg}(\mathcal{F})$ such that its unique deformed singularity $z_{t}$ near $z$ persists in being a center. We will prove that the foliation $\mathcal{F}_{t}$ has a first integral of the same type of $\mathcal{F}$. Using the arguments of the proof of this result we will give a lower bound for the maximum number of limit cycles of real polynomial differential equations of a fixed degree in the real plane.
\end{abstract}

Key words: Holomorphic foliation, limit cycle, center singularity.

\section{INTRODUCTION}

Consider the Hamiltonian equation

$$
\frac{d y}{d x}=-\frac{H_{x}}{H_{y}}
$$

in $\mathbb{C}^{2}$, where $H$ is a morse polynomial of degree $n+1$ in $\mathbb{C}^{2}$, i.e. the critical points of $H$ are nondegenerate with distinct images in $\mathbb{C}$. The above equation has $n^{2}$ singularities of center type (Morse type). Let also consider the perturbation

$$
\frac{d y}{d x}=-\frac{H_{x}+\epsilon P}{H_{y}+\epsilon Q}
$$

where $P$ and $Q$ are two polynomials of degree at most $n$. Ilyashenko in (Ilyashenko 1969) proves that if (1) has a center singularity for all $\epsilon$ then it must be again Hamiltonian. The objective of this 
article is to generalize Ilyashenko's result to foliations in $\mathbb{C} P(2)$ with a generic first integral of the type $\frac{F^{p}}{G^{q}}$.

Let $F$ and $G$ be two polynomials of degrees $a+1$ and $b+1$, respectively, in $\mathbb{C}^{2}, \frac{a+1}{b+1}=\frac{q}{p}$ and g.c.d. $(p, q)=1$. Consider the foliation $\mathcal{F}$ in $\mathbb{C} P(2)$ with the first integral

$$
\begin{gathered}
f: \mathbb{C} P(2) \backslash(\{F=0\} \cap\{G=0\}) \rightarrow \overline{\mathbb{C}} \\
f(x, y)=\frac{F(x, y)^{p}}{G(x, y)^{q}}
\end{gathered}
$$

In other words $\mathcal{F}$ is given by the 1 -form $p G d F-q F d G$. We will denote the foliation defined by the 1 -form $\omega$ by $\mathcal{F}(\omega)$. Note that the line at infinity $H_{\infty}=\mathbb{C} P(2) \backslash \mathbb{C}^{2}$ is neither pole nor zero component of $f$. A center singularity of $\mathcal{F}$ is a nondegenerate singularity of $\mathcal{F}$ with a local Morse integral i.e., around the singularity the foliation is given by $x^{2}+y^{2}=$ const. in some analytic coordinate system $(x, y)$. Therefore in the leaves around a center singularity there exists a nontrivial cycle which is called the Lefschetz vanishing cycle.

The points of $\{F=0\} \cap\{G=0\}$ are dicritical singularities of $\mathcal{F}$. If $F=0$ intersects $G=0$ transversally, these points are called the radial singularities of $\mathcal{F}$.

Let $\omega$ be a polynomial 1-form in $\mathbb{C}^{2}$. Considering $\omega$ as a meromorphic 1-form in $\mathbb{C} P(2)$, it has a pole of order $k$ along $H_{\infty}$. The degree of $\omega$ is defined to be $k-2$. This definition of degree has a difference up to one with the maximum of the degrees of the polynomials defining $\omega$ (see (Lins Neto and Scárdua 1997)).

We denote by $\mathcal{I}(a, b)$ the set of all integrable foliations of the above type, by $\mathcal{I}_{m}(a, b)$ the set of $\mathcal{F}=\mathcal{F}(p G d F-q F d G) \in \mathcal{I}(a, b)$ such that all singularities of $\mathcal{F}$ are centers or radials and the image of the centers under $\frac{F^{p}}{G^{q}}, 0$ and $\infty$ are distinct points in $\overline{\mathbb{C}}$ and by $\mathcal{F}(2, d)$ the space of foliations of degree $d$ in $\mathbb{C} P(2)$. It is easy to see that $\mathcal{I}(a, b) \subset \mathcal{F}(2, d)$, where $d=a+b$. For more information about holomorphic foliations the reader is referred to (Camacho and Sad 1987) and (Lins Neto and Scárdua 1997).

Proposition 1. $\mathcal{I}_{m}(a, b)$ is an open dense set in $\mathcal{I}(a, b)$.

Let $\mathcal{F}_{t} \in \mathcal{F}(2, d), t \in(\mathbb{C}, 0)$ be a holomorphic deformation of the foliation $\mathcal{F}=\mathcal{F}_{0}$ which has at least one center for all $t \in(\mathbb{C}, 0)$. If we know the structure of $\mathcal{F}$, for example the type of singularities, holonomy groups of some leaves and etc., then what can be said about the deformed foliation $\mathcal{F}_{t}$ ?

The foliation $\mathcal{F}$ has a finite number of singularities therefore, there exists a center $p \in \operatorname{sing}(\mathcal{F})$ and a sequence of centers $p_{t_{i}} \in \operatorname{sing}\left(\mathcal{F}_{t_{i}}\right)$ such that $p_{t_{i}} \rightarrow p$ when $t_{i} \rightarrow 0$. The deformed holonomy $h_{t}(x)$ related to the vanishing cycle around $p$, is the identity for $t=t_{i}$. Since $h_{t}$ is holomorphic in $t, h_{t}$ is the identity for all $t$. In particular, $p_{t}$ is a center for all $t \in(\mathbb{C}, 0)$.

Given an integrable foliation $\mathcal{F}(p G d F-q F d G) \in \mathcal{I}(a, b)$, let $p$ be one of the center singularities of $\mathcal{F}$ and let $\mathcal{F}_{t}=\mathcal{F}\left(p G d F-q F d G+t \omega_{1}+\right.$ h.o.t. $)$ be a holomorphic deformation 
of $\mathcal{F}$ in $\mathcal{F}(2, d)$, where $d=a+b$, such that its unique singularity $p_{t}$ near $p$ is still a center. Our principal theorem in this article is the following:

THEOREM 1. In the above situation, if $a+b>2$ then there exists an open dense subset $U$ of $\mathcal{I}(a, b)$ such that for all $\mathcal{F}(p G d F-q F d G) \in U$ and for all deformations $\mathcal{F}_{t}$ as before, $\mathcal{F}_{t}$ is also an integrable foliation. More precisely, there exist polynomials $F_{t}$ and $G_{t}$ such that $\mathcal{F}_{t}=\mathcal{F}\left(p G_{t} d F_{t}-q F_{t} d G_{t}\right)$, where $F_{t}$ and $G_{t}$ are holomorphic in $t$ and $F_{0}=F$ and $G_{0}=G$.

This theorem also says that the persistence of one center along the deformation implies the persistence of all other centers and radials. J.R. Muciño in (Muciño 1995) has also studied this problem in the case $p=q=1$. However, he has not obtained the result of Theorem 1 in this case, as we will explain later in $\$ 2$.

Example. Let $F$ and $G$ be generic conics. The pencil $F+t G=0$ contains a reducible fiber, say $F+t_{0} G=F_{1} F_{2}$, where $\operatorname{deg}\left(F_{1}\right)=\operatorname{deg}\left(F_{2}\right)=1$. Now $\mathcal{F}(F d G-G d F)=\mathcal{F}\left(F d\left(F_{1} F_{2}\right)-\right.$ $\left.F_{1} F_{2} d F\right)$ and this foliation admits the deformation

$$
\mathcal{F}\left(\lambda_{0} \frac{d F}{F}+\lambda_{1} \frac{d F_{1}}{F_{1}}+\lambda_{2} \frac{d F_{2}}{F_{2}}\right), 2 \lambda_{0}+\lambda_{1}+\lambda_{2}=0
$$

of degree two which is not integrable but has many center singularities. The reason for which Theorem 1 fails is that in this example we have reducible fibers. In fact only in the case $a+b \leq 2$ the foliation $\mathcal{F} \in \mathcal{I}_{m}(a, b)$ has reducible fibers. In the above example Theorem 3 of $\S 2$ is not true and hence Theorem 1 is not true also. From now on we will assume that $a+b>2$.

\section{IDEA OF THE PROOF OF THEOREM 1}

The proof of Theorem 1 lies in Theorems 2, 3, 4, and Proposition 2. First we consider the local situation of the theorem.

THEOREM 2. In the above situation, if the singularity of $\mathcal{F}_{t}$ near p persists in being a center then

$$
\int_{\delta} \frac{\omega_{1}}{F G}=0
$$

for all Lefschetz vanishing cycles $\delta$ in the leaves around the center $p$, where $\omega_{1}$ is the tangent vector of the deformation.

Definition 1. Let $\mathcal{F} \in \mathcal{F}(2, d)$ and let $\delta$ and $\delta^{\prime}$ be two closed cycles contained in two leaves of $\mathcal{F}$. We say that the cycle $\delta$ is $\mathcal{F}$-equivalent with $\delta^{\prime}$, if there is a continuous sequence of closed cycles $\delta_{t}, 0 \leq t \leq 1$ such that

- $\delta_{t}$ is a cycle in some leaf of $\mathcal{F}$;

- $\delta_{0}=\delta$ and $\delta_{1}=\delta^{\prime}$. 
A closed cycle $\delta$ is called a vanishing cycle if it is $\mathcal{F}$-equivalent with a zero cycle, which is a point $p$, in $(\mathbb{C} P(2) \backslash \sin g(\mathcal{F})) \cup\{p\}$, and is called a Lefschetz vanishing cycle if it is $\mathcal{F}$-equivalent with the singularity $p$ of a center in $(\mathbb{C} P(2) \backslash \sin g(\mathcal{F})) \cup\{p\}$. Note that here we consider a singularity as a leaf and therefore the cycles introduced around the centers satisfy this definition.

THEOREM 3. Let $\mathcal{F}(p G d F-q F d G) \in \mathcal{I}_{m}(a, b), a+b>2$, L be a regular leaf of $\mathcal{F}$ and $\delta$ be a Lefschetz vanishing cycle in $L$. Then the set of cycles in $L$ which are $\mathcal{F}$-equivalent with $\delta$ in $\mathbb{C} P(2) \backslash\left(\cup_{i=1}^{r} f^{-1}\left(c_{i}\right) \cup\{F=0\} \cup\{G=0\}\right)$ generate the first homology group of $L$ in the field of rational numbers.

The proof of above theorem uses arguments of the article (Lamotke 1981). By definition, the leaves of $\mathcal{F}(p G d F-q F d G)$ are the fibers of $\frac{F^{p}}{G^{q}}$ without the radial singularities. Therefore this theorem partially claims that the cycles around radial singularities are rational sums of cycles $\mathcal{F}$-equivalent with the cycle $\delta$. In the case $p=q=1, \mathcal{F}$ is a Lefschetz pencil and the mentioned fact is not explicitly stated in the literature. This has not any contradiction with Hard Lefschetz Theorem (4.1.3 p.29 (Lamotke 1981)). Note that the Hard Lefschetz Theorem is for compact fibers and not fibers with deleted radial singularities. Disconsideration of the above theorem in (Muciño 1995) has caused that the author of that paper has not obtained Theorem 1 in the case $p=q=1$. In fact he assumes the following unnecessary hypothesis: $\int \frac{\omega_{1}}{F G}$ vanishes over cycles around radial singularities. A deeper analysis of Lefschetz's argument (see (Lamotke 1981)) is needed to prove the above theorem in the case $p=q=1$.

Let $p$ be the center of $\mathcal{F}$ in Theorem 1 which persists in being a center after the deformation of $\mathcal{F}$. Applying Theorem 2 to the deformation

$$
\mathcal{F}_{t}: p \frac{d F}{F}-q \frac{d G}{G}+t \frac{\omega_{1}}{F G}+\text { h.o.t. }
$$

we obtain that

$$
\int_{\delta} \frac{\omega_{1}}{F G}=0
$$

for all vanishing cycles $\delta$ in the leaves around $p$ and by Theorem 3 we conclude that the equality (2) holds for all closed cycles $\delta$ in the leaves of the foliation $\mathcal{F}$, where the integral is defined. Partially we obtain that the residue of $\frac{\omega_{1}}{F G}$ on a leaf around a radial singularity is zero. The 1-form $\frac{\omega_{1}}{F G}$ is called a relatively exact 1 -form modulo the foliation $\mathcal{F}(p G d F-q F d G)$.

TheOREM 4. Let $\mathcal{F}(p G d F-q F d G) \in \mathcal{I}_{m}(a, b)$. Suppose that $\omega_{1}$ is a polynomial 1-form in $\mathbb{C}^{2}$ with deg $\left(\omega_{1}\right) \leq \operatorname{deg}(\mathcal{F})$ and $\frac{\omega_{1}}{F G}$ is relatively exact modulo $\mathcal{F}$. Then there is $(P, Q) \in \mathcal{P}_{a+1} \times \mathcal{P}_{b+1}$ such that $\omega_{1}$ has the form

$$
\omega_{1}=p G d P-q P d G+p Q d F-q F d Q
$$


Now let $\mathcal{M}(2, d)$ be the set of foliations of degree $d$ in $\mathbb{C} P(2)$ with at least one center. I have learned the statement and proof of the following proposition from A. Lins Neto.

Proposition 2. $\mathcal{M}(2, d)$ is an algebraic subset of $\mathcal{F}(2, d)$.

The next theorem identifies some irreducible components of $\mathcal{M}(2, d)$.

THeOREM 5. If $a+b>2$ then $\mathcal{I}(a, b)$ is an irreducible component of $\mathcal{M}(2, d)$, where $d=a+b$.

Proof. Since $\mathcal{I}(a, b)$ is parameterized by $\mathcal{P}_{a+1} \times \mathcal{P}_{b+1}, \mathcal{I}(a, b)$ is an irreducible variety. For any $\mathcal{F}(p G d F-q F d G) \in \mathcal{I}_{m}(a, b)$, we have seen in the theorems 2,3 and 4 that

$$
\begin{gathered}
{\left[\omega_{1}\right] \in T_{\mathcal{F}} \mathcal{M}(2, d) \Rightarrow} \\
\omega_{1}=p G d P-q P d G+p F d Q-Q d F,(P, Q) \in \mathcal{P}_{a+1} \times \mathcal{P}_{b+1} \Rightarrow \\
{\left[\omega_{1}\right] \in T_{\mathcal{F}} \mathcal{I}(a, b)}
\end{gathered}
$$

this and the fact that $\mathcal{I}(a, b) \subset \mathcal{M}(2, d)$ imply that

$$
T_{\mathcal{F}} \mathcal{M}(2, d)=T_{\mathcal{F}} \mathcal{I}(a, b), \forall \mathcal{F} \in \mathcal{I}_{m}(a, b)
$$

Since $\mathcal{I}_{m}(a, b)$ is an open dense subset of $\mathcal{I}(a, b)$, we conclude that $\mathcal{I}(a, b)$ is an irreducible component of $\mathcal{M}(2, d)$.

Theorem 1 is a direct consequence of the above Theorem.

\section{APPLICATION}

The idea of the following definition comes from the converse version of Theorem 2 .

Definition 2. Let $X$ be an irreducible component of $\mathcal{M}(2, d), \mathcal{F}\left(\omega_{0}\right) \in X$ and $p$ be a center of $\mathcal{F}$. There is a coordinate $(x, y)$ in a small neighbourhood $U$ of $p$ such that in this coordinate $p=(0,0)$ and

$$
\omega_{0}=g d(\tilde{f}), \tilde{f}=\frac{1}{2}\left(x^{2}+y^{2}\right)+\text { h.o.t., } g(0) \neq 0
$$

where $\tilde{f}$ and $g$ are holomorphic functions on $U$. Define $T_{\mathcal{F}}^{*} X$ as the set of all 1-forms $\left[\omega_{1}\right] \in$ $T_{\mathcal{F}} \mathcal{F}(2, d)$ such that

$$
\int_{\delta} \frac{\omega_{1}}{g}=0
$$

for all Lefschetz vanishing cycles in the leaves of $\mathcal{F}$ around $p$. By Theorem 2, we know that

$$
T_{\mathcal{F}} X \subset T_{\mathcal{F}}^{*} X
$$

$X$ is called a good irreducible component of $\mathcal{M}(2, d)$ if for a generic choice of $\mathcal{F} \in X$, the equality holds in (4). This definition does not depend on the choice of $g$. 
The process of the proof of Theorem 1 shows that $\mathcal{I}(a, b)$ is a good irreducible component of $\mathcal{M}(2, d)$.

Now let $X$ be an irreducible component of $\mathcal{M}(2, d), \mathcal{F}\left(\omega_{0}\right) \in X$ be a smooth point of $X \backslash(\mathcal{M}(2, d) \cap X)$ and $p$ be a center singularity of $\mathcal{F}$. Furthermore, if $\omega_{0}$ is a real 1 -form assume that the real foliation induced by $\omega_{0}$ has a real center at $p \in \mathbb{R}^{2}$.

THEOREM 6. There is a real polynomial differential equation of degree $d$ and with at least

$$
N=\operatorname{dim}\left(T_{\mathcal{F}} \mathcal{F}(2, d) / T_{\mathcal{F}}^{*} X\right)-1
$$

limit cycles in the real plane $\mathbb{R}^{2}$.

We obtain this fact by a small deformation of $\mathcal{F}\left(\omega_{0}\right)$, where $\omega_{0}$ is a real 1-form. Applying the above theorem to $\mathcal{I}(a, b)$ we have:

COROLlary 1. There is a real polynomial differential equation of degree $d$ and with at least

$$
\begin{cases}\frac{3}{4}\left(d^{2}+2 d-4 / 3\right) & \text { if } d \text { is even } \\ \frac{3}{4}\left(d^{2}+2 d-13 / 3\right) & \text { if } d \text { is odd }\end{cases}
$$

limit cycles.

\section{RESUMO}

Seja $\mathcal{F}$ uma folheação no espaço projetivo de dimensão dois e com integral primeira do tipo $\frac{F^{p}}{G^{q}}$, onde $F$ e $G$ são dois polinômios numa carta afim e $\frac{\operatorname{deg}(F)}{\operatorname{deg}(G)}=\frac{q}{p}$ e $g . c . d .(p, q)=1$. Seja $z$ um ponto crítico não degenerado de $\frac{F^{p}}{G^{q}}$ e $\mathcal{F}_{t}$ uma deformação de $\mathcal{F}$ no espaço das folheações de grau $\operatorname{deg}(\mathcal{F})$ tal que a singularidade deformada $z_{t}$ perto de $z$ ainda é um centro. Provamos que a folheação $\mathcal{F}_{t}$ tem uma integral primeira do mesmo tipo de $\mathcal{F}$. Usando os argumentos da demonstração desse resultado daremos uma cota inferior para o numero máximo de ciclos limites de uma equação differential de grau fixo no plano real.

Palavras-chave: folheação holomórfica, ciclo limite, singularidade do tipo centro.

\section{REFERENCES}

Camacho C and Sad P. 1987. Pontos Singulares de Equações Diferenciais Analíticas, $16^{\circ}$ Colóquio Brasileiro de Matemática.

ILyashenko YU. 1969. The Origin of Limit Cycles Under Perturbation of Equation $d w / d z=-R_{Z} / R_{w}$, where $\mathrm{R}(\mathrm{z}, \mathrm{w})$ is a Polynomial, Math. USSR, Sbornik, vol 7, No. 3.

Lamotкe K. 1981. The Topology of Complex Projective Varieties After S. Lefschetz, Topology, 20: 15-51.

Lins Neto A And Scárdua BA. 1997. Folheações Algébricas Complexas, $21^{\circ}$ Colóquio Brasileiro de Matemática.

MuciÑo JR. 1995. Deformations of holomorphic foliations having a meromorphic first integral, J reine angew Math 461: 189-219. 